\title{
BMJ Open Association between initial opioid prescription diagnosis type and subsequent chronic prescription opioid use in Rhode Island: a population-based cohort study
}

\author{
Benjamin D Hallowell (D) , ${ }^{1}$ Laura C Chambers, ${ }^{1}$ Luke Barre,${ }^{2}$ Nancy Diao, ${ }^{1}$ \\ Collette Onyejekwe, ${ }^{1}$ Alexandra Banks, ${ }^{3}$ Jeffery Bratberg, ${ }^{4}$ Heidi Weidele, ${ }^{1}$ \\ Samara Viner-Brown, ${ }^{1}$ James McDonald ${ }^{1}$
}

To cite: Hallowell BD, Chambers LC, Barre L, et al. Association between initial opioid prescription diagnosis type and subsequent chronic prescription opioid use in Rhode Island: a populationbased cohort study. BMJ Open 2022;12:e050540. doi:10.1136/ bmjopen-2021-050540

- Prepublication history for this paper is available online. To view these files, please visit the journal online (http://dx.doi. org/10.1136/bmjopen-2021050540).

Received 25 February 2021 Accepted 07 December 2021

Check for updates

(C) Author(s) (or their employer(s)) 2022. Re-use permitted under CC BY-NC. No commercial re-use. See rights and permissions. Published by BMJ.

${ }^{1}$ Rhode Island Department of Health, Providence, Rhode Island, USA

${ }^{2}$ Roger Williams Medical Center, Providence, Rhode Island, USA

${ }^{3}$ School of Public Health, Brown University, Providence, Rhode

Island, USA

${ }^{4}$ College of Pharmacy, University of Rhode Island, Kingston, Rhode Island, USA

\section{Correspondence to} Dr Benjamin D Hallowell; benjamin.hallowell@health. ri.gov

\section{ABSTRACT}

Objective To identify initial diagnoses associated with elevated risk of chronic prescription opioid use.

Design Population-based, retrospective cohort study. Setting State of Rhode Island.

Participants Rhode Island residents with an initial opioid prescription dispensed between 1 April 2019 and 31 March 2020.

Primary outcome measure Subsequent chronic prescription opioid use, defined as receiving 60 or more days' supply of opioids in the 90 days following an initial opioid prescription.

Results Among the 87055 patients with an initial opioid prescription, 3199 (3.7\%) subsequently became chronic users. Patients who become chronic users tended to receive a longer days' supply, greater quantity dispensed, but a lower morphine milligram equivalents on the initial opioid prescription. Patients prescribed an initial opioid prescription for diseases of the musculoskeletal system and connective tissue (adjusted OR (aOR): $5.9,95 \% \mathrm{Cl}: 4.7$ to 7.6 ), diseases of the nervous system (a0R: $6.3,95 \% \mathrm{Cl}$ : 4.9 to 8.0 ) and neoplasms (aOR: $5.6,95 \% \mathrm{Cl}: 4.2$ to 7.5 ) had higher odds of subsequent chronic prescription opioid use, compared with a referent group that included all diagnosis types with fewer than 15 chronic opioid users, after adjusting for confounders.

Conclusions By focusing interventions and prescribing guidelines on specific types of diagnoses that carry a high risk of chronic prescription opioid use and diagnoses that would benefit equally or more from alternative management approaches, states and healthcare organisations may more efficiently decrease inappropriate opioid prescribing while improving the quality of patient care.

\section{INTRODUCTION}

In developed countries, pain is one of the most common reasons people seek medical care and managing this pain appropriately can be clinically challenging. ${ }^{1}$ An analysis of the National Health Interview Survey by
Strengths and limitations of this study

- This study included all Rhode Island residents with an initial opioid prescription dispensed by a licensed retail pharmacy during the 12-month study period, which decreases selection bias and provides more generalisable results.

- A high percentage (36\%) of initial opioid prescriptions had a missing diagnosis code during the analysis period, which may bias our results.

- Identifying logical, simple and accurate ways to collapse International Classification of Disease codes, 10th revision (ICD-10), into meaningful groups is difficult.

the Centers for Disease Control and Prevention estimated that approximately 50 million Americans are burdened with chronic pain, and of those, 19.6 million have pain that frequently impacts their life or work activities. ${ }^{2}$ While prescription opioids may be effective at relieving pain, they fail to alleviate the underlying cause of pain, and the evidence supporting their effectiveness at relieving chronic pain is controversial. ${ }^{3}$ Additionally, chronic prescription opioid use can cause dependence in some individuals, which may lead to addiction, overdose, and/or death.

To help define safe prescribing practices, many studies have tried to identify factors associated with prolonged opioid use following specific diagnoses or procedures, including childbirth, major surgeries or chronic conditions. ${ }^{4-6}$ While many studies have identified patient-level risk factors associated with chronic opioid use among those with a specific diagnosis (eg, higher doses, history of substance abuse, anxiety or tobacco use), none have attempted to identify initial 
diagnoses associated with elevated risk of chronic opioid use overall. ${ }^{5-7}$

Previous regulations limiting opioid prescribing have been effective at reducing inappropriate or unsafe opioid prescribing practices. ${ }^{8}$ Properly characterising the underlying diagnoses and procedures that resulted in chronic prescription opioid use could inform targeted prescribing guidelines, particularly if those underlying diagnoses and procedures can be managed effectively with non-opioid therapies. To inform these knowledge gaps, we leveraged population-based controlled substance prescribing data to estimate the association between the diagnosis type on the initial opioid prescription and subsequent chronic prescription opioid use.

\section{METHODS}

\section{Study design and population}

We conducted a population-based retrospective cohort study utilising data from the Rhode Island Prescription Drug Monitoring Programme (PDMP). Our cohort included all Rhode Island residents with an initial opioid prescription dispensed by a retail pharmacy with a controlled substance registration between 1 April 2019 and 31 March 2020.

We identified opioid prescriptions using the American Hospital Formulary Service Pharmacologic-Therapeutic Classification Code (TCC) associated with the National Drug Code of each product in the IBM Micromedex RED BOOK, including opiate agonists (TCC 28:08.08), opiate partial agonists (TCC 28:08.12) and tramadol products (TCC 28:08.92.00.50). We excluded antitussive and antidiarrhoeal medications and buprenorphine products that were only approved by the US Food and Drug Administration for medication-assisted treatment for opioid use disorder as of 30 July 2020 due to our interest in opioids prescribed for pain management. We defined an initial opioid prescription as either (1) the patient's first opioid prescription or (2) an opioid prescription that started at least 60 days after the patient's previous opioid prescription ended. When defining initial opioid prescriptions, we utilised prescriptions filled on/after 1 January 2019 to ensure there was a 'look-back' of at least 90 days for all patients. For patients with more than one initial opioid prescription during the analysis period, we randomly selected one initial prescription for inclusion in the analysis to maintain independence of observations.

In 2018, Rhode Island began requiring prescribers to include the primary International Classification of Disease codes, 10th revision (ICD-10), on all prescriptions for a controlled substance. ${ }^{9}$ In this study, our primary exposure was the diagnosis type associated with the primary ICD-10 code on the initial opioid prescription. We classified ICD-10 codes into 20 major diagnostic groups based on ICD-10 code chapters and separately presented specific ICD-10 codes associated with at least 15 chronic opioid users. Our primary outcome was subsequent chronic prescription opioid use, defined as $\geq 60$ days' supply of opioids dispensed in the 90 days following the fill date of an individual's initial opioid prescription (including the days' supply of the initial prescription). ${ }^{10}$ When monitoring subsequent chronic opioid prescription use, we utilised prescription data through 1 July 2020 to ensure at least 90 days of follow-up following the initial opioid prescription for all patients.

We excluded veterinary prescriptions based on an indicator for veterinary prescriptions, an animal name field and the degrees listed with the prescriber's last name (ie, DVM or VMD). Additionally, we excluded initial opioid prescriptions filled by pharmacies for which ICD-10 codes were missing for more than $80 \%$ of their dispensed opioid prescriptions due to concerns about bias among the ICD-10 codes that were entered. We defined unique patients based on the first five letters of the last name, the first three letters of the first name and the date of birth on each prescription.

\section{Statistical analysis}

We estimated the association between the diagnosis type on the initial opioid prescription and subsequent chronic prescription opioid use using logistic regression, adjusting for potential confounders. The diagnosis categories with fewer than 15 chronic opioid users were collapsed and used as the reference group. Patients with missing diagnosis type were excluded from the model. We selected the following potential confounders a priori: age (continuous) and sex. Characteristics of the initial prescription (eg, type of opioid, days' supply, quantity dispensed, daily morphine milligram equivalents (MME)) were not considered confounders because they are determined by the prescriber based on the diagnosis and, thus, are in the causal pathway between the diagnosis type and subsequent chronic opioid use. While other sociodemographic and clinical factors likely impact chronic opioid use (eg, socioeconomic status), these variables are not recorded in the Rhode Island PDMP database. To understand the potential impact of missing diagnosis category information, we compared the characteristics of initial opioid prescriptions with and without a diagnosis.

All analyses were conducted in SAS V.9.4 (Cary, North Carolina, USA).

\section{Patient and public involvement}

No patient or public involvement.

\section{RESULTS}

Between 1 April 2019 and 31 March 2020, there were a total of 113398 initial opioid prescriptions dispensed to 99129 unique Rhode Island residents. Of these, 98883 initial opioid prescriptions to 87055 unique people were dispensed by pharmacies with $<80 \%$ of initial opioid prescriptions missing an ICD-10 code. After randomly selecting one initial opioid prescription for each of the 10294 patients with more than one during this period, 
Table 1 Baseline characteristics of Rhode Island residents dispensed initial opioid prescriptions, 1 April 2019 to 31 March 20201

Subsequent chronic prescription opioid use ${ }^{\star}$

\begin{tabular}{llll} 
Overall & Yes & No \\
\cline { 2 - 3 } $\begin{array}{l}\mathrm{N}=87055 \\
\mathrm{n}(\%) \dagger\end{array}$ & $\mathrm{N}=3199$ & $\mathrm{~N}=83856$ \\
$\mathrm{n}(\%) \dagger$
\end{tabular}

\section{Characteristics}

Patient Characteristics

Age

Sex

Female

Male

Unknown

Payment method

Private insurance

Medicare

Medicaid

Cash

Workers' compensation

Unknown

Prescription Characteristics

Opioid type

Oxycodone

Hydrocodone

Tramadol

Codeine

Morphine

Hydromorphone

Buprenorphine (pain)§

Methadone

Fentanyl

Tapentado

Opium

Oxymorphone

Butorphanol

Pentazocine

Dihydrocodeine

Meperidine

Initial opioid prescription

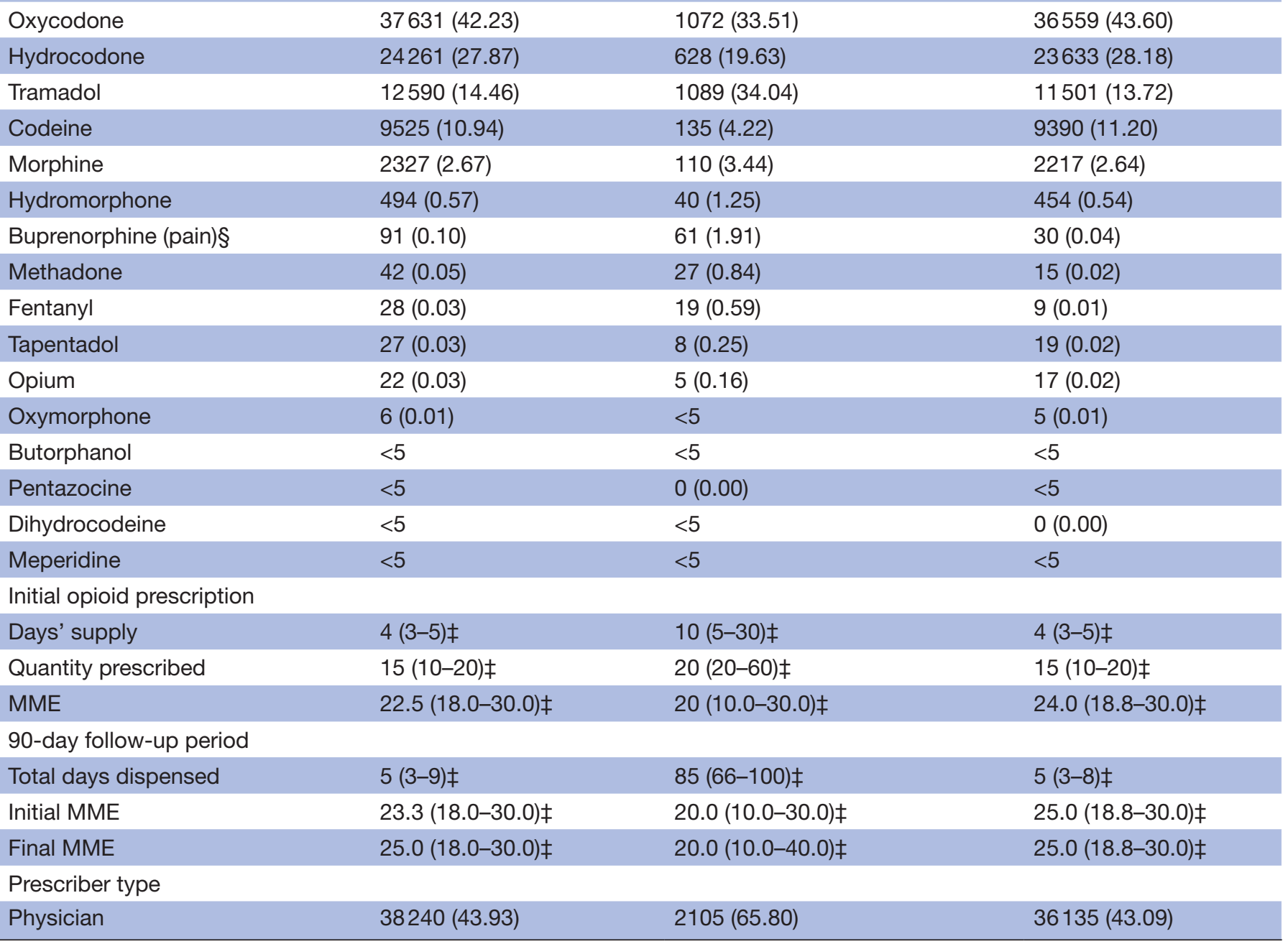

Continued 


\begin{tabular}{llll}
\hline & & \multicolumn{2}{l}{ Subsequent chronic prescription opioid use* } \\
\cline { 2 - 4 } & Overall & Yes & No \\
\cline { 2 - 4 } Characteristics & $\begin{array}{l}\mathbf{N}=\mathbf{8 7 0 5 5} \\
\mathbf{n ~ ( \% ) \dagger}\end{array}$ & $\begin{array}{l}\mathbf{N}=3199 \\
\mathbf{n}(\%) \dagger\end{array}$ & $\mathbf{N}=83856$ \\
$\mathbf{n}(\%) \dagger$
\end{tabular}

*Chronic prescription opioid use defined as $\geq 60$ days' supply of opioids dispensed in the 90 days following the fill date of an individual's initial opioid prescription (including the days' supply of the initial prescription).

†Unless otherwise specified.

$\ddagger$ Median (IQR).

$\S$ This analysis excluded buprenorphine products only FDA-approved for medication-assisted treatment of opioid use disorder. IInitial opioid prescriptions defined as either the patient's first opioid prescription or an opioid prescription that started at least 60 days after the patient's previous opioid prescription ended. For patients with more than one initial opioid prescription during this period, we randomly selected one for inclusion in this analysis.

MME, morphine milligram equivalent.

there were 87055 initial opioid prescriptions dispensed to 87055 unique people in the final study population.

\section{Baseline characteristics of initial opioid prescriptions}

The median age of patients receiving an initial opioid prescription was 53 years ( $\mathrm{IQR}=36-66)$, and a majority $(57.9 \%)$ of patients were female. Most patients paid for the initial opioid prescription with private insurance $(60.3 \%)$, followed by Medicare $(14.9 \%)$ and Medicaid (12.4\%). The most common types of opioids prescribed on the initial prescription were oxycodone (42.2\%), hydrocodone $(27.9 \%)$, tramadol $(14.5 \%)$ and codeine $(10.9 \%)$. The median days' supply prescribed, and quantity prescribed were 4 days $(\mathrm{IQR}=3-5)$ and 15 units $(\mathrm{IQR}=10-20)$, respectively. The median daily MME was $22.5(\mathrm{IQR}=18.0-30.0$, table 1$)$. Most of the initial opioid prescriptions were prescribed by physicians (43.9\%).

Patients with and without subsequent chronic prescription opioid use following the initial prescription differed on all baseline characteristics compared, except for sex and the median MME on their initial opioid prescription. Of note, patients with versus without subsequent chronic prescription opioid use following the initial prescription tended to be older (median: 63 vs 53 years), more likely to have paid for their initial opioid prescription with Medicare $(27.5 \%$ vs $14.4 \%$ ), and less likely to have paid with private insurance $(44.3 \%$ vs $60.9 \%)$. Additionally, patients with versus without subsequent chronic use were somewhat more likely to have been prescribed tramadol $(34.0 \%$ vs $13.7 \%)$, hydromorphone $(1.3 \%$ vs $0.5 \%)$ or buprenorphine pain $(1.9 \%$ vs $0.0 \%)$ products; and had a longer median days' supply (10 vs 4 days) and greater quantity dispensed (20 vs 15 units). Finally, patients with versus without subsequent chronic use were somewhat more likely to have been prescribed their initial opioid prescription by a physician (65.8\% vs $43.1 \%$ ) or an advanced nurse ( $16.9 \%$ vs $13.6 \%$ ) rather than a dentist $(0.7 \%$ vs $17.0 \%)$ or a physician assistant $(11.6 \%$ vs $15.2 \%)$.

Overall, $32574(36.27 \%)$ patients were missing diagnosis code information on their initial opioid prescription. The most common diagnosis codes for the remaining 55481 patients included diseases of the musculoskeletal system and connective tissue $(\mathrm{n}=15525,17.8 \%)$, diseases of the nervous system $(\mathrm{n}=8377,9.6 \%)$ and diseases of the digestive system $(\mathrm{n}=7552,8.7 \%$; table 2$)$. Notably, patients with unknown versus known diagnosis type were somewhat more likely to pay for their initial opioid prescription with cash $(11.2 \%$ vs $8.2 \%)$, to have received hydrocodone (32.6\% vs $25.2 \%)$ or codeine $(15.2 \%$ vs $8.5 \%)$ products on their initial opioid prescription, and to have received their initial opioid prescription from a dental professional $(29.0 \%$ vs $9.2 \%$, table 3$)$.

\section{Opioid prescribing during the 90-day follow-up period}

In the 90 days following the start of the initial opioid prescription, the daily MME increased slightly from a median of 23.3 MME (IQR=18.0-30.0) on the first day of opioids prescribed to 25.0 ( $\mathrm{IQR}=18.0-30.0)$ on the last day of opioids prescribed. Overall, patients were prescribed a median of 5 days $(\mathrm{IQR}=3-9)$ of opioids total during the 90-day follow-up period.

Among the 87055 patients with an initial opioid prescription, 3199 (3.7\%) subsequently had chronic prescription opioid use per our definition. Similarly, among the 55481 patients with an initial opioid prescription that included a known diagnosis type, 2305 (4.2\%) subsequently had chronic prescription opioid use (table 4). When compared with patients prescribed an initial opioid prescription in the referent group (ie, all diagnosis categories with fewer than 15 chronic opioid users), those with diseases of the musculoskeletal system and connective tissue (adjusted OR (aOR): 5.9, 95\% CI: 
Table 2 Diagnosis type on initial opioid prescriptions dispensed to Rhode Island residents, 1 April 2019 to 31 March 2020*

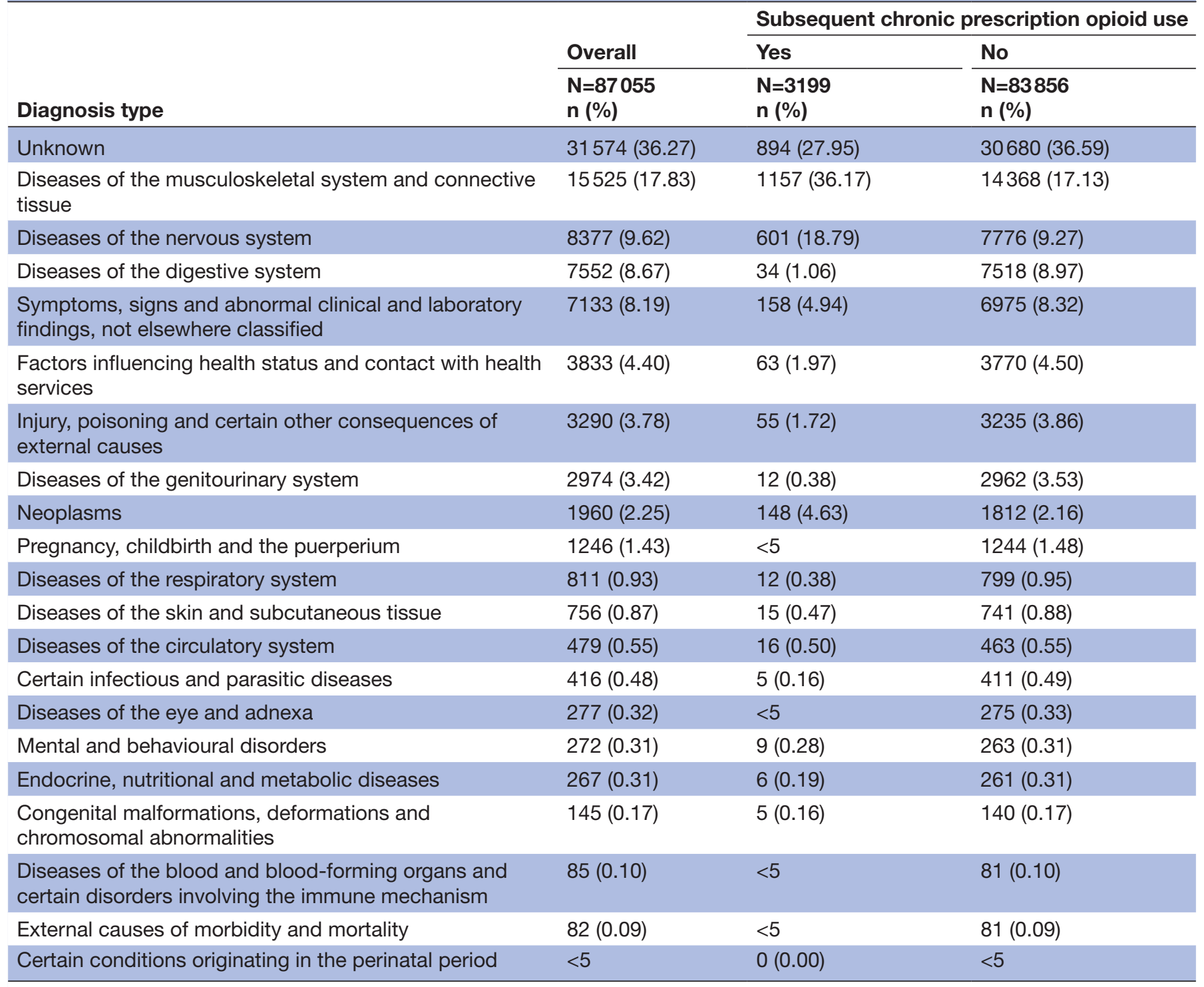

*Initial opioid prescriptions were defined as either the patient's first opioid prescription or an opioid prescription that started at least 60 days after the patient's previous opioid prescription ended. For patients with more than one initial opioid prescription during this period, we randomly selected one for inclusion in this analysis.

4.7 to 7.6), diseases of the nervous system (aOR: 6.3, 95\% CI: 4.9 to 8.0 ) and neoplasms (aOR: $5.6,95 \%$ CI: 4.2 to 7.5) had higher odds of subsequent chronic prescription opioid use after adjusting for age and sex, and had an elevated percentage of patients with chronic use when compared with the overall average $(7.5 \%, 7.2 \%$ and $7.6 \%$, respectively, when compared with $1.0 \%)$.

When looking at the specific ICD-10 codes with at least 15 patients with subsequent chronic prescription opioid use, pain not elsewhere classified (G89; 592/2305, $25.7 \%$ ), dorsalgia (M54; 477/2305, 20.7\%), pain, unspecified (R52; 95/2305, 4.1\%) and other joint disorders not elsewhere classified (M25; 94/2305, 4.1\%) had the highest number of chronic users (table 5). Notably, specific diagnoses that had a relatively high percentage of patients who progressed to chronic use following the initial opioid prescription included: intraoperative and postprocedural complications and disorders of musculoskeletal system, not elsewhere classified (31/89, 34.8\%); polyosteoarthritis $(38 / 205,18.5 \%)$; rheumatoid arthritis (15/84, 17.9\%); and spondylosis $(69 / 407,17.0 \%)$.

\section{DISCUSSION}

In the 12-month study period, nearly $10 \%$ of Rhode Island residents received at least one initial opioid prescription, of whom $3.7 \%$ went on to become chronic prescription opioid users based on our study definition. Tramadol, hydromorphone and buprenorphine pain products were more often prescribed on the initial opioid prescription to people who became chronic prescription opioid users. Moreover, chronic users tended to receive a longer days' 
Table 3 Characteristics associated with unknown diagnosis type on initial opioid prescriptions dispensed to Rhode Island residents, 1 April 2019 to 31 March $2020 \S$

\begin{tabular}{|c|c|c|}
\hline Characteristics & $\begin{array}{l}\text { Known } \\
\text { diagnosis type } \\
\mathrm{N}=55481 \\
\mathrm{~N}(\%)^{*}\end{array}$ & $\begin{array}{l}\text { Unknown } \\
\text { diagnosis type } \\
\mathrm{N}=31574 \\
\mathrm{~N}(\%)^{\star}\end{array}$ \\
\hline \multicolumn{3}{|l|}{ Patient characteristics } \\
\hline Age & $54(37-66) \dagger$ & $52(34-65) \dagger$ \\
\hline \multicolumn{3}{|l|}{ Sex } \\
\hline Female & 33007 (59.49) & $17392(55.08)$ \\
\hline Male & $22474(40.51)$ & $14178(44.90)$ \\
\hline Unknown & $0(0.0)$ & $<5$ \\
\hline \multicolumn{3}{|l|}{ Payment method } \\
\hline Private insurance & $33777(60.88)$ & $18714(59.27)$ \\
\hline Medicare & $8358(15.06)$ & $4590(14.54)$ \\
\hline Medicaid & $6861(12.37)$ & $3968(12.57)$ \\
\hline Cash & $4552(8.20)$ & $3527(11.17)$ \\
\hline Workers' compensation & $241(0.43)$ & $192(0.61)$ \\
\hline Unknown & $1692(3.05)$ & $583(1.85)$ \\
\hline \multicolumn{3}{|l|}{ Prescription characteristics } \\
\hline \multicolumn{3}{|l|}{ Opioid type } \\
\hline Oxycodone & $26422(47.62)$ & $11209(35.50)$ \\
\hline Hydrocodone & $13955(25.15)$ & $10306(32.64)$ \\
\hline Tramadol & $8417(15.17)$ & $4173(13.22)$ \\
\hline Codeine & $4719(8.51)$ & $4806(15.22)$ \\
\hline Morphine & $1483(2.67)$ & $844(2.67)$ \\
\hline Hydromorphone & $314(0.57)$ & $180(0.57)$ \\
\hline Buprenorphine (pain) $\ddagger$ & $70(0.13)$ & $21(0.07)$ \\
\hline Methadone & $32(0.06)$ & $10(0.03)$ \\
\hline Fentanyl & $25(0.05)$ & $<5$ \\
\hline Tapentadol & $16(0.03)$ & $11(0.03)$ \\
\hline Opium & $18(0.03)$ & $<5$ \\
\hline Oxymorphone & $<5$ & $<5$ \\
\hline Butorphanol & $<5$ & $<5$ \\
\hline Pentazocine & $0(0.00)$ & $<5$ \\
\hline Dihydrocodeine & $<5$ & $0(0.00)$ \\
\hline Meperidine & $<5$ & $<5$ \\
\hline \multicolumn{3}{|l|}{ Chronic opioid use } \\
\hline Yes & $472(0.85)$ & $175(0.55)$ \\
\hline No & 55009 (99.15) & $31399(99.45)$ \\
\hline \multicolumn{3}{|l|}{ Initial opioid prescription } \\
\hline Days' supply & $5(3-5) \dagger$ & $4(3-5) \dagger$ \\
\hline Quantity prescribed & $18(10-20) \dagger$ & $15(10-20) \dagger$ \\
\hline Daily MME & $25.0(18.8-30.0) \dagger$ & $22.5(18.0-30.0) \dagger$ \\
\hline \multicolumn{3}{|l|}{ 90-day follow-up period } \\
\hline Total days prescribed & $5(3-5) \dagger$ & $5(3-7) \dagger$ \\
\hline Initial MME & $25.0(18.8-30.0) \dagger$ & $22.5(18.0-30.0) \dagger$ \\
\hline Final MME & $25.0(18.8-30.0) \dagger$ & $22.5(18.0-30.0) \dagger$ \\
\hline \multicolumn{3}{|l|}{ Prescriber type } \\
\hline Physician & $26083(47.01)$ & $12157(38.50)$ \\
\hline Physician assistant & 8954 (16.14) & $4123(13.06)$ \\
\hline
\end{tabular}

Continued
Table 3 Continued

\begin{tabular}{cll}
\hline & $\begin{array}{l}\text { Known } \\
\text { diagnosis type } \\
\mathbf{N}=\mathbf{5 5 4 8 1} \\
\mathbf{N}(\%)^{*}\end{array}$ & $\begin{array}{l}\text { Unknown } \\
\text { diagnosis type } \\
\mathbf{N}=\mathbf{3 1 5 7 4} \\
\mathbf{N}(\%)^{*}\end{array}$ \\
\hline Advanced nurse & $8458(15.24)$ & $3515(11.13)$ \\
Dental professional & $5101(9.19)$ & $9161(29.01)$ \\
Unknown & $6885(12.41)$ & $2618(8.29)$ \\
\hline
\end{tabular}

*Unless otherwise specified.

†Median (IQR).

$\ddagger$ This analysis excluded buprenorphine products that were only FDAapproved for medication-assisted treatment of opioid use disorder. §Initial opioid prescriptions were defined as either the patient's first opioid prescription or an opioid prescription that started at least 60 days after the patient's previous opioid prescription ended. For patients with more than one initial opioid prescription during this period, we randomly selected one for inclusion in this analysis.

MME, morphine milligram equivalent.

supply, greater quantity dispensed, but a lower MME on the initial opioid prescription. Physicians and advanced nurses more often prescribed the initial opioid prescription of patients who become chronic prescription opioid users. Overall, initial opioid prescriptions were most often prescribed for diseases of the musculoskeletal system and connective tissue, diseases of the nervous system and diseases of the digestive system, and these diagnoses were strongly associated with subsequent chronic prescription opioid use. Among ICD-10 codes with at least 15 chronic users, the percentage of patients who went on to develop chronic prescription opioid use following the initial prescription ranged from $1.9 \%$ to $34.8 \%$ by the specific ICD-10 code.

While prior work has focused on chronic prescription opioid use following specific diagnoses or procedures, our population-based study evaluated chronic prescription opioid use among all patients receiving initial opioid prescriptions in Rhode Island. ${ }^{4-6}$ Despite differences in methodology, other studies estimating the frequency of chronic prescription opioid use after childbirth $(1.7 \%-2.2 \%)$, dental procedures $(2.4 \%-4.1 \%)$, elective surgeries $(3.0 \%-8.0 \%)$ or cancer treatment $(8.3 \%)$ identified a similar percentage of patients with chronic use compared with our study of all patients receiving initial opioid prescriptions $(3.67 \%) .^{4-711-14}$ In the absence of similar population-based studies, these comparisons suggest that the percentage of patients experiencing subsequent chronic prescription opioid use in our study was generally similar to what might be expected based on prior work. While other studies often define chronic use as one or more claims for opioid prescriptions 0-90 and 90-180 days after the procedure/diagnosis, we defined chronic use as at least 60 days' supply of opioids dispensed in the 90 days following the fill date of an individual's initial opioid prescription (including the days' supply of the initial prescription). ${ }^{57910}$ We used this relatively strict definition of chronic prescription opioid in an attempt to exclude patients who intermittently used opioids and to focus our analysis on opioid prescribing patterns most 
Table 4 Association between the diagnosis type on the initial opioid prescription and subsequent chronic prescription opioid use among Rhode Island residents, 1 April 2019 to 31 March 2020†,

\begin{tabular}{|c|c|c|c|}
\hline \multirow[b]{2}{*}{ Diagnosis type } & \multicolumn{2}{|c|}{$\begin{array}{l}\text { Subsequent chronic } \\
\text { prescription opioid use }\end{array}$} & \multirow[b]{2}{*}{ Adjusted OR $(95 \% \mathrm{Cl}) \ddagger$} \\
\hline & $\begin{array}{l}\text { No } \\
\mathrm{N}=53176 \\
\mathrm{n}(\text { row \%) }\end{array}$ & $\begin{array}{l}\text { Yes } \\
\mathrm{N}=2305 \\
\text { n (row \%) }\end{array}$ & \\
\hline Diseases of the musculoskeletal system and connective tissue & $14368(92.55)$ & $1157(7.45)$ & $5.94(4.68-7.56)$ \\
\hline Diseases of the nervous system & $7776(92.83)$ & $601(7.17)$ & $6.26(4.90-8.01)$ \\
\hline $\begin{array}{l}\text { Symptoms, signs and abnormal clinical and laboratory findings, } \\
\text { not elsewhere classified }\end{array}$ & $6975(97.78)$ & $158(2.22)$ & $1.96(1.48-2.60)$ \\
\hline Factors influencing health status and contact with health services & $3770(98.36)$ & $55(1.67)$ & $1.34(0.96-1.89)$ \\
\hline $\begin{array}{l}\text { Injury, poisoning and certain other consequences of external } \\
\text { causes }\end{array}$ & $3235(98.33)$ & $55(1.67)$ & $1.38(0.97-1.96)$ \\
\hline
\end{tabular}

Significant adjusted odds ratios where $\mathrm{p}<0.05$ are bolded

*Initial opioid prescriptions defined as either the patient's first opioid prescription or an opioid prescription that started at least 60 days after the patient's previous opioid prescription ended. For patients with more than one initial opioid prescription during this period, we randomly selected one for inclusion in this analysis.

†Chronic prescription opioid use defined as $\geq 60$ days' supply of opioids dispensed in the 90 days following the fill date of an individual's initial opioid prescription (including the days' supply of the initial prescription).

$\ddagger$ Adjusted for potential confounders selected a priori: age (continuous) and sex.

$\S O t h e r$ includes all initial opioid prescriptions for diagnosis types with 15 or less chronic users, including: certain conditions originating in the perinatal period, certain infectious and parasitic diseases, congenital malformations, deformations and chromosomal abnormalities, diseases of the blood and blood-forming organs and certain disorders involving the immune mechanism, diseases of the eye and adnexa, external causes of morbidity and mortality, pregnancy, childbirth and the puerperium, mental and behavioural disorders, endocrine, nutritional and metabolic diseases, diseases of the skin and subcutaneous tissue, diseases of the respiratory system and diseases of the genitourinary system.

likely to represent chronic use and carry the greatest risk of opioid addiction. ${ }^{15}$

Unfortunately, the Rhode Island PDMP only recently started collecting ICD-10 codes on controlled substance prescriptions, and as such, we do not know if the percentage of patients who developed chronic prescription opioid use following an initial prescription has changed over time. Additionally, it is possible that the 'legacy population' of long-term prescription opioid users in Rhode Island began their chronic use through different diagnoses than those reported here, particularly given changes in prescribing patterns over the past 5-10 years. The Rhode Island Department of Health, in collaboration with other sister state agencies and communitybased organisations, has launched several interventions that may have reduced the chronic prescription opioid use over time, including one-on-one targeted prescriber education on responsible prescribing, appropriate regulation regarding prescribing, and provider reimbursement for the use of non-opioid pain management strategies. ${ }^{91617}$

A primary benefit of categorising diagnosis type through ICD-10 codes in this population-based study is the ability to systematically review opioid prescribing patterns among patients with different diseases or sources of pain. Previous studies have shown the dangers of inappropriate prescribing, but effectively targeting interventions and prescribing guidelines to minimise risk of chronic opioid use while appropriately managing pain is challenging. ${ }^{18}$ Our study identified a few diagnosis types on initial opioid prescriptions that are associated with greater odds of subsequent chronic prescription opioid use in Rhode Island, which allows our state to prioritise interventions that target specific practice areas. For instance, we found that diseases of the musculoskeletal system and connective tissue were highly associated with subsequent chronic prescription opioid use, and initial opioid prescriptions with ICD-10 codes for polyosteoarthritis, rheumatoid arthritis, spondylosis and intraoperative/postprocedural complications and disorders of the musculoskeletal system had a particularly high percentage of patients develop chronic use after initiation. Given that many patients with musculoskeletal pain can improve over time independent of treatment, prior work has suggested that non-pharmacological treatments be utilised first (eg, physical therapy, acupuncture, massages), followed by or with anti-inflammatory medications, and if patients are still experiencing pain, 
Table 5 ICD-10 codes on initial opioid prescriptions with the highest number of subsequent chronic prescription opioid users, Rhode Island residents-1 April 2019 to 31 March 2020*

\begin{tabular}{|c|c|c|}
\hline \multirow[b]{3}{*}{ Diagnosis } & \multicolumn{2}{|c|}{ Subsequent chronic prescription opioid use } \\
\hline & $\begin{array}{l}\mathrm{N}=2305 \\
\mathrm{n}(\text { row \%) }\end{array}$ & $\begin{array}{l}\mathrm{N}=53176 \\
\mathrm{n}(\text { row \%) }\end{array}$ \\
\hline & Yes & No \\
\hline Pain, not elsewhere classified (G89) & $592(7.86)$ & $6087(92.14)$ \\
\hline Dorsalgia (M54) & $477(9.75)$ & $4416(90.25)$ \\
\hline Pain, unspecified (R52) & $95(2.27)$ & $4081(97.73)$ \\
\hline Other joint disorder, not elsewhere classified (M25) & $94(4.76)$ & $1880(95.24)$ \\
\hline Spondylosis (M47) & $69(16.95)$ & $338(83.05)$ \\
\hline Thoracic, thoracolumbar, and lumbosacral intervertebral disc disorders (M51) & $67(13.76)$ & $420(86.24)$ \\
\hline Osteoarthritis of knee (M17) & $59(7.11)$ & $771(92.89)$ \\
\hline Other and unspecified soft tissue disorders, not elsewhere classified (M79) & $58(5.48)$ & $1001(94.52)$ \\
\hline Other and unspecified osteoarthritis (M19) & $50(12.17)$ & $361(87.83)$ \\
\hline Other spondylopathies (M48) & $42(8.11)$ & $476(91.89)$ \\
\hline Polyosteoarthritis (M15) & $38(18.54)$ & $167(81.46)$ \\
\hline $\begin{array}{l}\text { Intraoperative and postprocedural complications and disorders of } \\
\text { musculoskeletal system, not elsewhere classified (M96) }\end{array}$ & $31(34.83)$ & $58(65.17)$ \\
\hline Abdominal and pelvic pain (R10) & $23(2.03)$ & $1108(97.97)$ \\
\hline Oseoarthritis of hip (M16) & $22(5.46)$ & $381(94.54)$ \\
\hline Malignant neoplasm of bronchus and lung (C34) & $22(14.29)$ & $132(85.71)$ \\
\hline Presence of other functional implants (Z96) & $17(3.83)$ & $427(96.17)$ \\
\hline Other deforming dorsopathies (M43) & $15(8.77)$ & $156(91.23)$ \\
\hline Other rheumatoid arthritis (M06) & $15(17.86)$ & $69(82.14)$ \\
\hline All other ICD-10 codes & $592(1.88)$ & 30847 (98.12) \\
\hline
\end{tabular}

*Initial opioid prescriptions were defined as either the patient's first opioid prescription or an opioid prescription that started at least 60 days after the patient's previous opioid prescription ended. For patients with more than one initial opioid prescription during this period, we randomly selected one for inclusion in this analysis.

prescription opioids. ${ }^{19} 20$ Importantly, as the pathophysiology of musculoskeletal diseases is inflammatory, a prescription opioid to block pain is not clinically appropriate, and if pharmacological therapy is necessary, antiinflammatory medications may be more efficacious than opioids. ${ }^{21}$ This is true for diagnoses of polyosteoarthritis, rheumatoid arthritis and spondylosis, which are generally inflammatory in nature, and depending on the diagnosis standard treatment guidelines recommend treatment with non-steroidal anti-inflammatory drugs, corticosteroids, muscle relaxants, steroids, disease-modifying antirheumatic drugs, biologics, anti-seizure medications, warming therapy, exercise and/or antidepressants rather than opioid pain management. ${ }^{22}$ By focusing interventions and prescribing guidelines on specific types of diagnoses that carry a high risk of chronic opioid use and benefit equally or more for alternative management approaches, states and healthcare organisations may efficiently decrease inappropriate opioid prescribing while improving the quality of patient care. Our study suggests that interventions focused on appropriate management of musculoskeletal conditions, including polyosteoarthritis, rheumatoid arthritis and spondylosis, may be particularly beneficial given the overall association with chronic use and the high percentage of patients who develop chronic use after an initial opioid prescription for one of these diagnoses.

This study can serve as an example for other states looking to undertake a similar analysis of new chronic prescription opioid use in their population and highlights the added value of collecting ICD-10 codes associated with prescriptions in PDMPs. Replicating this study in other regions could inform the standard of care for pain management and hopefully lead to safer prescribing patterns.

A major strength of this study is the inclusion of all opioid prescriptions dispensed to Rhode Island residents by licensed retail pharmacies for the 12-month study period through the Rhode Island PDMP. The use of a large, population-based study decreases selection bias and provides more generalisable results, although we are not able to identify specific subpopulations with unique risk profiles using this approach. Additionally, our restrictive definition of chronic prescription opioid use 
likely minimised the inclusion of intermittent prescription opioid users in this group, improving the specificity of our outcome. Finally, this study demonstrates the utility of collecting ICD-10 codes in PDMPs, as this allows for consideration of the reason for prescriptions in population-based epidemiologic studies. This highlights for us the advantage of increasing ICD-10 reporting. As reporting increases, this dataset provides a way to systematically identify the diagnoses associated with all opioid prescriptions dispensed to Rhode Island residents.

Despite the advantages of having ICD-10 codes in the Rhode Island PDMP, the largest limitation of this study was the high percentage (36\%) of opioid initiate prescriptions with a missing diagnosis code during the analysis period. Unfortunately, we are not able to determine whether the prescriber neglected to provide an ICD-10 code on the prescription, or the pharmacy neglected to include the ICD-10 code when reporting to the PDMP. Although initial opioid prescriptions with and without ICD-10 codes were significantly different on all characteristics compared, our sample size was large, and the most meaningful difference was that prescriptions missing ICD-10 codes were more often written by dental professionals. It may be useful to support dental professionals in the reporting of ICD-10 codes, including sharing the results of these types of studies, to improve the completeness of reporting. Additionally, this analysis is limited to opioids dispensed by retail pharmacies and does not include those dispensed at hospitals or by Veterans Affairs, which may modify our results. Finally, due to variation in the specificity of ICD-10 codes, identifying logical, simple and accurate ways to collapse them into meaningful groups is difficult. Improved systematic methods for categorising ICD-10 codes into clinically meaningful groups would be useful for future epidemiologic studies.

\section{CONCLUSIONS}

Despite nearly $10 \%$ of Rhode Island residents receiving an initial opioid prescription in the 12-month study period, $3.7 \%$ went on to become chronic prescription opioid users based on our study definition. Diseases of the musculoskeletal system and connective tissue, diseases of the nervous system and neoplasms carried a high risk of subsequent chronic opioid use. For diagnoses in these groups that would benefit equally or more from alternative management approaches, we can create targeted interventions and prescribing guidelines to efficiently decrease inappropriate opioid prescribing while improving the quality of patient care.

\section{Acknowledgements The authors would like to thank Victoria Ayers for her guidance in drafting this report.}

Contributors $\mathrm{BH}, \mathrm{LCC}, \mathrm{AB}$ and HW made substantial contributions to the conception of the work, the acquisition, analysis and interpretation of data, drafting the work and revising it critically for important intellectual content and gave final approval of the version to be published and agreement to be accountable for all aspects of the work in ensuring that questions related to the accuracy or integrity of any part of the work are appropriately investigated and resolved. LB, ND, CO,
JB, SV-B and JM made substantial contributions to the conception of the work, revising it critically for important intellectual content, and gave final approval of the version to be published and agreement to be accountable for all aspects of the work in ensuring that questions related to the accuracy or integrity of any part of the work are appropriately investigated and resolved. BH accepts full responsibility for the finished work and/or the conduct of the study, had access to the data, and controlled the decision to publish.

Funding The authors have not declared a specific grant for this research from any funding agency in the public, commercial or not-for-profit sectors.

\section{Competing interests None declared.}

Patient and public involvement Patients and/or the public were not involved in the design, or conduct, or reporting or dissemination plans of this research.

Patient consent for publication Not applicable.

Ethics approval This study was deemed exempt by the Institutional Review Board of the Rhode Island Department of Health (IRB approval number: 2019-30).

Provenance and peer review Not commissioned; externally peer reviewed.

Data availability statement Data from the Rhode Island Prescription Drug Monitoring Program can be obtained by submitting a request to the Drug Overdose Surveillance Program at the Rhode Island Department of Health. Please visit: https://ridoh-overdose-surveillance-rihealth.hub.arcgis.com/.

Open access This is an open access article distributed in accordance with the Creative Commons Attribution Non Commercial (CC BY-NC 4.0) license, which permits others to distribute, remix, adapt, build upon this work non-commercially, and license their derivative works on different terms, provided the original work is properly cited, appropriate credit is given, any changes made indicated, and the use is non-commercial. See: http://creativecommons.org/licenses/by-nc/4.0/.

ORCID iD

Benjamin D Hallowell http://orcid.org/0000-0002-6943-9615

\section{REFERENCES}

1 Finley CR, Chan DS, Garrison S, et al. What are the most common conditions in primary care? systematic review. Can Fam Physician 2018;64:832-40.

2 Dahlhamer J, Lucas J, Zelaya C, et al. Prevalence of Chronic Pain and High-Impact Chronic Pain Among Adults - United States, 2016. MMWR Morb Mortal Wkly Rep 2018;67:1001-6.

3 Rosenblum A, Marsch LA, Joseph H, et al. Opioids and the treatment of chronic pain: controversies, current status, and future directions. Exp Clin Psychopharmacol 2008;16:405-16.

4 Soneji N, Clarke HA, Ko DT, et al. Risks of developing persistent opioid use after major surgery. JAMA Surg 2016;151:1083-4.

5 Peahl AF, Dalton VK, Montgomery JR, et al. Rates of new persistent opioid use after vaginal or cesarean birth among US women. JAMA Netw Open 2019;2:e197863.

6 Vitzthum LK, Riviere P, Sheridan P, et al. Predicting persistent opioid use, abuse, and toxicity among cancer survivors. J Natl Cancer Inst 2020;112:720-7.

7 Brummett CM, Waljee JF, Goesling J, et al. New persistent opioid use after minor and major surgical procedures in US adults. JAMA Surg 2017;152:e170504.

8 Barre L, Oliver B, Alexander-Scott N, et al. Impact of state regulations on initial opioid prescribing behavior in Rhode island. $R$ I Med J 2019;102:24-6.

9 RIDOH. Pain assessment (216-RICR-20-15-2), 2018. Available: https://rules.sos.ri.gov/regulations/part/216-20-15-2 [Accessed 18 Nov 2020].

10 Fulton-Kehoe D, Von Korff M, Mai J, et al. Surveillance of opioid prescribing as a public health intervention: Washington state Bree collaborative opioid metrics. J Public Health Manag Pract 2020;26:206-13.

11 Harbaugh CM, Lee JS, Chua K-P, et al. Association between long-term opioid use in family members and persistent opioid use after surgery among adolescents and young adults. JAMA Surg 2019;154:e185838.

12 O'Hara NN, Pollak AN, Welsh CJ, et al. Factors associated with persistent opioid use among injured workers' compensation claimants. JAMA Netw Open 2018;1:e184050.

13 Brescia AA, Waljee JF, Hu HM, et al. Impact of prescribing on new persistent opioid use after cardiothoracic surgery. Ann Thorac Surg 2019;108:1107-13. 
14 Stark N, Kerr S, Stevens J. Prevalence and predictors of persistent post-surgical opioid use: a prospective observational cohort study. Anaesth Intensive Care 2017;45:700-6.

15 Inacio MCS, Hansen C, Pratt NL, et al. Risk factors for persistent and new chronic opioid use in patients undergoing total hip arthroplasty: a retrospective cohort study. BMJ Open 2016;6:e010664.

$16 \mathrm{RIDOH}$. Opioid overdose prevention and reporting (216-RICR-2020-5), 2014. Available: https://rules.sos.ri.gov/regulations/part/21620-20-5 [Accessed 18 Nov 2020].

17 RIDOH. Pain management, opioid use and the registration of Distributors of controlled substances in Rhode Island (216-RICR-2020-4), 2020. Available: https://rules.sos.ri.gov/regulations/part/21620-20-4 [Accessed 18 Nov 2020].
18 Barre L, Gallo J, McDonald JV. Review of disciplinary actions regarding controlled substances, Rhode island 2012-2017. J Med Regul 2019;105:22-7.

19 George SZ, Goode AP. Physical therapy and opioid use for musculoskeletal pain management: competitors or companions? Pain Rep 2020;5:e827.

20 Qaseem A, Wilt TJ, McLean RM, et al. Noninvasive treatments for acute, subacute, and chronic low back pain: a clinical practice guideline from the American College of physicians. Ann Intern Med 2017;166:514-30.

21 Schnitzer TJ, Elliott AM, LM B. Update on guidelines for the treatment of chronic musculoskeletal pain. Clin Rheumatol 2006;25(Suppl 1):22-9.

22 Bullock J, Rizvi SAA, Saleh AM, et al. Rheumatoid arthritis: a brief overview of the treatment. Med Princ Pract 2018;27:501-7. 